\title{
Einleitung zu "Form und Bedeutung" \\ Kompositionelle, funktionale und autonome Syntax
}

\author{
VolKMAR ENGERER \\ Staatsbibliothek Århus, Dänemark
}

\section{EINLEITUNG ZUR EINLEITUNG}

Der Zusammenhang zwischen Syntax als Lehre von der Form sprachlicher Ausdrücke mit dem Satz als höchster Komplexitätsebene und dem, was die Ausdrücke (bis hin zum Satz) ausdrücken oder „bedeuten“, ist in der Sprachtheorie grundlegend, sowohl was den Grammatikbegriff und das Verständnis der Syntax als Formwissenschaft als auch die Konzeption der jeweiligen semantischen Theorie angeht. ${ }^{1}$ Man kann in der linguistischen Literatur meiner Meinung nach zwei Strategien unterscheiden, das Verhältnis von formorientierter Syntax und Bedeutungsaspekten zu thematisieren. Man findet als erste Hauptgruppe im eigentlichen Sinn theorieinteressierte Ansätze, welche, ausgehend von einem jeweils syntaktischen oder semantischen (oder, übergeordnet, informationstheoretischen) Standbein, an relativ beschränktem Datenmaterial die jeweiligen Zusammenhänge, Abhängigkeiten oder jeweils autonomen Züge im SyntaxSemantik-Bereich untersuchen.

Die hier vorgelegten Beiträge sind als solche Studien zu verstehen. Alle versuchen, in abgegrenzten grammatischen Teilbereichen (z.B. Argumentselektion, Syntax des Verbalkomplexes, Adverbialsyntax, syntaktische Relationen, Negation usw.) Aussagen darüber zu formulieren, wie syntaktische Strukturierungsprinzipien mit semantischen interagieren: Ist es die Bedeutung von Konstituenten, welche die syntaktische Position von Ausdrücken bestimmt? Laut Haider, in diesem Band, ja, wenn von Adverbialen die Rede ist. Oder ist es die einzelsprachliche Syntax, welche die Bedingungen für die semantische Interpretation absteckt? Wiederum, laut Haider, ja, wenn es um einzelsprachliche (oder sprachtypologisch) bedingte Restriktionen bei wh-Bewegungen geht. Viele der hier gesammelten Beiträge zeugen davon, dass das Syntax-SemantikVerhältnis je nach Datenbereich und Sprachtyp verschieden ausgestaltet sein kann. Ich werde in dieser kleinen Einführung in Bezug auf die semantische Komponente vorläufig drei syntaktische Datenbereiche unterscheiden, die, mehr oder weniger explizit, in den hier vorgelegten Studien aufscheinen.

Eine Art Gegenpol zu solchen Studien sind gesamtgrammatische Darstellungen sowie Gesamtdarstellungen der Syntax einer Einzelsprache X (Grammatik der Xen Sprache, Syntax des Xen). Solcherlei Unternehmen auferlegen der jeweiligen Konzeption des Form-Bedeutungszusammenhangs ganz besondere (und strenge, möchte ich meinen) Restriktionen, die sich in grammatischen Einzelstudien nicht in dem Masse in den 
Vordergrund drängen. In meinem eigenen Beitrag zu diesem Sammelband (s. Engerer, in diesem Band) lege ich den Versuch einer Analyse des Syntax-Semantik-Verhältnisses in grammatischen Gesamtdarstellungen vor, im ersten Schritt von zwei ausgewählten Syntaxen des Deutschen. Dies ist ein Projekt, das ich in späteren Einzelanalysen fortführen werde.

Ich möchte in der Einleitung zu diesem Band einige Beispiele des ersten Typs diskutieren, also solcher Ansätze, die das Problem des Syntax-Semantik-Zusammenhangs direkt zum Gegenstand haben. Diese Überlegungen werden, wie ich hoffe, einige der hier versammelten Beiträge in ein etwas schärferes Licht stellen.

\section{Der Wettstreit zwischen Syntax und Semantik}

\subsection{Formelle und funktionale Sprachwissenschaft}

Das Verhältnis zwischen Syntax und Semantik wird oft, z.B. von Vikner, als sprachtheoretischer Wettstreit zweier konkurrierender linguistischer Ansätze (und damit verbunden linguistischer Schulen und Traditionen) interpretiert (Vikner 2004). Das formelle sprachwissenschaftliche Paradigma betrachtet sprachliche Form als etwas, das unabhängig von der kommunikativen Funktion beschrieben werden kann. Auf der anderen Seite stehen die Vertreter des funktionalen Zugangs, die ihren Ausgangspunkt in der kommunikativen Funktion sprachlicher Einheiten sehen, die ihrerseits entscheidend die sprachliche Form bestimmen kann (Vikner 2004: 13). Diesen beiden Paradigmen entsprechen tendenziell wiederum bestimmte Grammatiktypen, man denke an „formelle Grammatiken“ strukturalistischer oder generativer Prägung auf der einen Seite sowie „funktionale Grammatiken“ im Stil der Prager Schule auf der anderen Seite, hierunter auch formale Grammatiktheorien wie die Relationale Grammatik oder die Lexical Functional Grammar. ${ }^{2}$ Man hat also auf beiden Seiten eine bunte Liste linguistischer Schulen, Methoden sowie formaler und informaler Theorien, denen man ihr jeweiliges Verhältnis zur Bedeutungsebene sowie kommunikativen Funktion sprachlicher Formen nicht immer auf den ersten Blick ansieht.

Die formelle und die funktionale Argumentation demonstriert Vikner an folgendem einfachen Fragesatz:

\section{1) Hvor vil David gerne bo?}

Der $h v$-Ausdruck $h v o r$ an der Satzspitze entspricht einem Lokaladverbial, das, auf generativistische Manier, in einem früheren Stadium der Ableitungskette seinen Platz rechts neben dem Hauptverb bo hatte und von dort seine Reise an den Satzanfang angetreten hat. ${ }^{3} \mathrm{Vgl} .(2)$.

\section{2) $\quad\left[{ }_{-}\right]_{\mathrm{i}}$ vil David gerne bo $[\text { hvor }]_{\mathrm{i}}$ ?}

An diesem Beispiel zeigt Vikner, dass Satzerststellung von $h v o r$ eine generelle Eigenschaft von $h v$-Elementen ist (es betrifft alle $h v$-Ausdrücke), die auch eingebettete Objekte umfasst ([Hvor mange bøger] har ...) und nicht einmal auf Fragesätze beschränkt ist 
(vgl. Vikner 2004: 13). Funktionale Erklärungen dieser Stellungsregel wie „Wichtige Elemente zuerst!“ oder „Referenzidentische Ausdrücke nebeneinander!“ greifen daher zu kurz - sie erfassen nach Vikner nicht den formellen, rein grammatischen Charakter der Umstellungsregel.

Den formellen und den funktionalen Zugang bei der Analyse sprachlicher Form sieht Vikner (2004: 14) als miteinander vereinbar an; welcher von den beiden Sprachvariation besser erklärt, ist letzten Endes eine empirische Frage. Dies führt Vikner auch zur Zurückweisung dessen, was er „extremen Funktionalismus“ und „extremen Formalismus“4 nennt, also die Verabsolutierung der beiden Ansätze (Vikner 2004: 14).

\subsection{Die Autonomiehypothese}

Vikners Diskussion von formellen und funktionalen Betrachtungsweisen in der Sprachwissenschaft gründet in dem bekannten Disput um die sogenannte Autonomiehypothese, die v.a. von Generativisten in den 80er Jahren verstärkt vertreten wurde. ${ }^{5}$ Auf dem Hintergrund einer kognitiven Konzeption von Grammatik als ein System sprachbezogener mentaler Repräsentationen (Chomsky 1981; Fanselow/Felix 1990: 66) wird die Existenz einer einheitlich strukturierten kognitiven Semantikkomponente in Abrede gestellt, jedenfalls in dem Sinne, dass sie dezidiert dem Sprachwissen zuzurechnen sei (Fanselow/Felix 1990: 66). Eigenständige und geschlossene sprachliche Systeme bilden nach dieser These nur die Syntax und Phonologie, die kurz und bündig als „Grammatik“ oder „formale Kompetenz" bezeichnet werden (Fanselow/Felix 1990: 66). ${ }^{6}$

Was heißt nun „autonom“? Die Syntax und die Phonologie sind autonom nach gängiger Ansicht in dem Sinne, dass sie Gesetzmäßigkeiten aufweisen, „[...] die sich in keinerlei anderen Wissensdomänen wiederfinden und daher auch nicht auf andere kognitive Struktursysteme reduzierbar sind." (Fanselow/Felix 1990: 67) Diese Gesetzmäßigkeiten „[...] sind nur mit eigenständigen grammatischen Kategorien formulierbar und erklären sich auch nur aus grammatischen Prinzipien, nicht aber etwa aus pragmatischen Prinzipien oder den Gesetzmäßigkeiten der allgemeinen menschlichen Problemlösungskompetenz." (Fanselow/Felix 1990: 67)

Die Autonomiethese in dieser Form besagt natürlich nicht, wie auch Vikner (2004) hervorhebt, „[...] dass zwischen syntaktischen Strukturen und semantischfunktionalen Gegebenheiten kein Zusammenhang bestehe." (Fanselow/Felix 1990:70) Selbstverständlich gibt es, wie die autonomen Syntaktiker einräumen, „semantische Effekte“ syntaktischer Prozesse, aber ebenso oft sind syntaktische Regeln nicht in einleuchtender Weise mit einer Bedeutungsvariation verbunden (Fanselow/Felix 1990: 70). Diese abgeschwächte Autonomiehypothese steht gut in Einklang mit Vikners These vom formellen sprachwissenschaftlichen Paradigma, das sich nicht auf einen extremen Formalismus (s.o.) versteift, wie aus folgendem Zitat hervorgeht: 
„Die These der Autonomie der Syntax besagt ja keineswegs, dass Grammatik nichts mit Semantik oder Pragmatik $\mathrm{zu}$ tun hat. Autonomie bedeutet ausschließlich, dass die grammatischen Regeln nicht auf semantische bzw. pragmatische Phänomene reduzierbar sind, d.h. es gibt keine semantische/pragmatische Gesetzmäßigkeit, aus denen die grammatischen Regeln notwendigerweise folgen." (Fanselow/ Felix 1990: 71)

Vertreter der syntaktischen Autonomiethese (und ich werde im Folgenden die Phonologie beiseite lassen) akzeptieren natürlich, unter Hinweis auf die Arbeiten von Montague, ${ }^{7}$ das (allen) einleuchtende Faktum, „[...] dass zwischen Syntax und Semantik ein sehr enges Verhältnis besteht, bzw. dass die Struktur syntaktischer Repräsentationen weitgehend von semantischen Gesetzmäßigkeiten bestimmt wird.“ (Fanselow/Felix 1990: 86) Diese Kernaufgabe der Grammatik, nämlich den Aufbau größerer syntaktischer Einheiten (Konstituenten) aus kleineren systematisch an den Aufbau entsprechender semantischer Einheiten zu koppeln, ist für Anhänger der Autonomiethese, so scheint es, eine periphere Funktion der Syntax/Grammatik - und rechtfertigt alleine nicht das Ansetzen einer (autonomen) syntaktischen Komponente (Fanselow/Felix 1990: 86). Es ist vielmehr die kleine Menge semantisch gesehen funktionsloser, „rein syntaktischer“ formeller Regeln, die ein autonomes und formelles Syntaxmodul konstituieren. Zudem konkurrieren in den meisten Fällen (in den meisten Datenbereichen) semantische und syntaktische Erklärungen, was es nötig macht, die semantisch bedingten syntaktischen Prozesse herauszufiltern. Was bleibt, ist der syntaktische hard-core-Bereich - eben autonom.

\subsection{Ein Beispiel: syntaktische Kontrolle}

Fanselow/Felix demonstrieren dies am bekannten Beispiel der Kontrollkonstruktionen (Fanselow/Felix 1990: 87-90). ${ }^{8}$

\section{3a) Ich verspreche ihm, zu kommen}

3b) Ich bitte ihn, zu kommen

In (3a) liegt Subjektkontrolle (der syntaktisch nicht realisierte Agensreferent des Infinitivsatzes ist identisch mit dem Subjektsreferenten des einbettenden Matrixsatzes - ich), in (3b) liegt Objektkontrolle vor (hier ist es der Objektreferent von ihn, der das Agens des Infinitivsatzes liefert). Die erste Schwierigkeit einer syntaktischen Erklärung der Variation von Subjekt- und Objektkontrolle liegt darin, dass es Verben wie glauben, vgl. (4), gibt, die beides zulassen, was auf eine verbsemantisch bedingte, also nichtsyntaktische Steuerung der Kontrolleigenschaften hindeutet (Fanselow/Felix 1990: 87).

\section{4) Ich glaubte ihm, gestern abend besoffen gewesen zu sein}

Dazu kommt, dass die Kontrolleigenschaften von der Grammatik der abhängigen zuInfinitiv-Verbgruppe abhängt. Passivierung (5a) und Modalisierung (5b) bewirken Mehrdeutigkeit, d.h. beide Lesarten sind möglich. 
5a) Ich versprach ihm/bat ihn, eingeladen zu werden

5b) Ich versprach ihm, nicht in die Schule gehen zu müssen

Bei passender Wahl des Kontextes favorisiert versprechen Objektkontrolle (6a), was sogar ohne Modalverb möglich ist (6b).

\section{6a) Der Chefarzt versprach dem Patienten, diese Nacht schlafen zu können}

6b) Der Chefarzt versprach dem Patienten, diese Nacht zu überstehen

Die Kontrollverhältnisse hängen also deutlich sowohl vom Matrixverb als auch vom Inhalt des abhängigen $z u$-Infintivsatzes ab (Fanselow/Felix 1990: 88). Fanselow/Felix geben im Folgenden (Fanselow/Felix 1990: 88f) die „wirkliche“ Erklärung der eben besprochenen Kontrollphänomene, die, wie schnell zu sehen ist, auf der sprechaktbedingten Verteilung der Aktanten bei den Verben versprechen und bitten beruht: Der Versprechende - das Subjekt im Matrixsatz - ist im Regelfall auch das Agens des Versprochenen, weil nur eigene Handlungen, und nicht die Dritter, versprochen werden können (das liegt in den Sprechaktbedingungen von Kommissiven wie Versprechen). Dagegen ist es im Kontext des Bittens der Objektreferent des Matrixsatzes, der vorzugsweise als Agens im Infinitivsatz fungiert (und der denotiert wiederum das, worum gebeten wird - man bittet um die Handlungen anderer). ${ }^{9}$ Zudem können wissensbasierte Schlussprozesse die sprechakttheoretischen Standardverteilungen der Aktanten aufheben, wie die obigen Beispiele (6) zeigen. Das Wissen um eine enge Verbindung von Patient-sein und der Möglichkeit, die Nacht nicht zu überstehen, löst einen kausalen interpretativen Zwischenschritt in Bezug auf den Referenten des syntaktischen Subjekts im Matrixsatz aus, der als der Verursacher des Sachverhaltes, dass der Patient die Nacht übersteht, interpretiert wird.

Autonome Syntax ist dies nicht - was aber könnte als syntaktisch-autonomer Rest der Grammatik von Kontrollkonstruktionen übrigbleiben? Fanselow/Felix (1990: 89) nennen in Anlehnung an Chomsky (1969) das syntaktische Datum, dass „[...] in derartigen Infinitivsätzen stets nur das Subjekt, nicht aber das Objekt fehlen darf“. Darüber hinaus ist pluraler Bezug des mitverstandenen Infinitivsubjekts auf zwei vorhandene Mitspieler des Matrixverbs ausgeschlossen, ebenso wenig wie der Referent des Subjekts im Infinitivsatz zwei Satzknoten höher liegen darf (Fanselow/Felix 1990: 89f). Dies sind also die mageren Kandidaten für wirklich autonome syntaktische Beschränkungen.

In ihrer Auseinandersetzung mit dem Funktionalismus, hier v.a. der Funktionalen Grammatik, wiederholen Fanselow/Felix (1990: 93ff) noch einmal ihr Argument, dass es einen autonomen Bereich syntaktischer Gesetzmäßigkeiten gibt, der nicht auf Funktionales (Semantik, Pragmatik, Textstruktur) zurückzuführen ist (Fanselow/Felix 1990: 93), und belegen dies mit einer weiteren Reihe von Beispielen. 


\subsection{Das Syntax-Semantik-Interface: der kompositionelle Aspekt}

Nach dem bisher Gesagten scheint es mir mindestens drei Typen des Verhältnisses zwischen Syntax und Semantikzu geben. Der erste-autonome-TypbetrifftDatenbereiche, deren einzige „Erklärung“ in formellen Regeln liegt, die keine Entsprechungen im funktionalen (semantischen, pragmatischen oder textstrukturellen) Bereich aufweisen. Die eigentliche Erklärung liegt somit jenseits struktureller Gegebenheiten, sie wird im Postulat eines angeborenen formellen Sprachorgans gesucht (s.a. Pinker 1996). Der zweite Typ deckt Datenbereiche, in denen syntaktisch-autonome und funktional motivierte Beschreibungen nebeneinander existieren und vielleicht sogar miteinander konkurrieren. Der dritte Typ, sozusagen der Gegenpol zur autonomen Syntax, ist der reguläre Typ. Hier ist die Rede von syntaktischen Strukturen, die regulär den Aufbau der entsprechenden semantischen Strukturen determinieren (oder umgekehrt). Auf diesen Typ möchte ich nun zu sprechen kommen.

Der systematische Zusammenhang von Syntax und Semantik kann etwas technisch als Interfaceproblematik (im engeren Sinne) beschrieben werden (Enç 1988). Wie muss so ein Interface beschaffen sein? Um einen Satz $S$ interpretieren zu können, muss das Interface $I$ die Interpretationen seiner Teile, i.A. lexikalischer Elemente, liefern (Enç 1988: 239). Des weiteren muss ein Austausch bzw. ein In-Beziehung-Setzen syntaktischer und semantischer Einheiten gewährleistet sein. Enç (1988: 239f) demonstriert dies an syntaktischen Funktionen (Subjekt, Objekt, ...) und semantischen Rollen (Agens, Patiens, ....). In den beiden Sätzen

\section{7a) Mary kissed John \\ 7b) John kissed Mary}

ist es ausschließlich die (syntaktische) Information, welche Konstituente Subjekt und welche Objekt ist, die eine korrekte Zuordnung der verbspezifischen semantischen Rollen - und damit auch eine korrekte Formulierung des Bedeutungsunterschieds der beiden Sätze - erlaubt. Von solchen bedeutungsrelevanten syntaktischen Merkmalen sind solche zu unterscheiden, die keine semantischen Reflexe haben. Enç (1988: 240) argumentiert, dass der Aktivsatz in (7a) zusammen mit der passivierten und topikalisierten Version (8a/ b, s.u.) dieselben Wahrheitsbedingungen aufweisen, was nicht nur für die vorliegenden Sätze, sondern für alle grammatisch äquivalenten lexikalischen Besetzungen gilt (und damit eine systematische Eigenschaft von syntaktischen Konstruktionen ist).

\section{8a) John was kissed by Mary}

8b) John, Mary kissed

Dies heißt wiederum, dass ein Syntax-Semantik-Interface $I$ zwischen semantisch relevanten (wahrheitwertverändernden) und semantisch irrelevanten (die Wahrheitsbedingungen nicht berührenden) syntaktischen Umstellungsprozessen unterscheiden können muss. Weiterhin muss die Verteilung der semantischen Rollen in (7a) (Mary - ,Küssende', John - ,Geküsster') auch in den syntaktischen Varianten (8a/b) 
konstant gehalten werden, und dies ungeachtet der Tatsache, dass die beiden Mitspieler Mary und John andere syntaktische Positionen als Subjekt und Objekt einnehmen (by Mary - Präpositionalphrase als Adverbial, John - topikalisiertes Element in Vorvorfeldposition, akzentuell abgehoben). Eine Möglichkeit, diese Konstanz zu sichern, besteht darin, eine syntaktisch definierte Ableitungsbeziehung zwischen Aktivsatz auf der einen Seite und passivierter bzw. topikalisierter Konstruktion auf der anderen Seite zu definieren. Beim Übergang von der Ausgangsstruktur im Aktiv werden dann die auf der Subjekt- bzw. Objektfunktion definierten semantischen Rollen (Küssende/r-Geküsste/r) auf die neuen, abgeleiteten Positionen übertragen. Dies ist, in der einen oder anderen Schattierung, wohl das Standardvorgehen in der grammatischen Beschreibung des Passivs (z.B. Engel 1988: 454ff sowie sehr ausgeprägt Dudengrammatik 1998: 174ff). ${ }^{10}$

Diese hier angedeutete Konzeption des Syntax-Semantik-Interface läuft auf eine wahrheitsfunktionale, dem Kompositionalitätsprinzip verpflichtete Fassung des Zusammenhangs von Syntax und Semantik hinaus (s.a. Enç 1988: 240). ${ }^{11}$ Relevant für die Semantik sind solche syntaktische Prozesse und Regeln, welche im Sinne einer 1-zu-1-Entsprechung zum Aufbau semantischer Strukturen beitragen. Semantische Strukturen sind wahrheitswertbasiert, d.h. sie liefern die formalen Bedingungen für die Wahrheit eines Ausdrucks (s.a. Lewis 1976). Liegt nun eine syntaktische Regel vor, die kein offenbares wahrheitswertrelevantes Pendant auf der semantischen Seite aufweist (wie die Passivierungs- oder Topikalisierungsregel, s.o.), ist sie, aus der Sicht des Semantikers, eine „bloß syntaktische Regel“, wobei offen bleibt, ob ihre Anwendung textstrukturell-pragmatisch bedingt (wie im Falle der Passivierung und Topikalisierung) oder dem Bereich der autonomen Syntax zuzurechnen ist. Also auch aus dieser Sicht, aus der Sicht der wahrheitsfunktionalen Semantik, ergibt sich eine ähnliche Strukturierung der syntaktischen Datenbereiche in drei Typen wie oben vorgeschlagen: Phänomene der a) kompositionalen Syntax sind solche, die systematisch eine Kopplung an wahrheitswertrelevante semantische Regeln zulassen. ${ }^{12}$ Die b) autonome Syntax hat solche Regeln zum Gegenstand, die rein formeller Art sind und keine weitere Motivierung darüber hinaus aufweisen (sie sind einfach kognitive Beschränkungen). Die c) funktionale Syntax umfasst alle syntaktischen Phänomene minus a) und b), d.h. solche syntaktischen Regeln und Prozesse, die wahrheitsfunktional irrelevant, jedoch nicht rein formeller Natur sind - und daher eine wie immer geartete funktionale, pragmatisch-textstrukturelle, Erklärung herausfordern. Beispiele hierfür sind die bereits erwähnte Passivierung und Topikalisierung, die beide, zusammen mit anderen Wortumstellungsregeln, die Anpassung der Thema-Rhema-Struktur eines Satzes an den vorangehenden Kontext sichern.

Syntax ist also, in Bezug auf die semantische Komponente, nicht nur autonom, nur funktional oder nur kompositional. Es ist meiner Meinung nach die Inhomogenität syntaktischer Phänomene, welche unterschiedliche und komplementäre Konzeptionen des Syntax-Semantik-Verhältnisses fordert. Die obige Dreiteilung in kompositionale, autonome und funktionale syntaktische Daten ist schematisch, aber spiegelt nach meinem Dafürhalten doch gut die sich noch immer als Konkurrenten betrachtenden 
Fraktionen im Lager der Syntaktiker und Semantiker wider.

\subsection{Was fehlt}

Meine kurze Skizze berücksichtigt nicht die mehr sprachwissenschaftshistorischen Bestimmungen des Syntax-Semantik-Verhältnisses, die seit jeher, schon mit Beginn der Antike, prominente Gegenstände sprachwissenschaftlicher und sprachphilosophischer Betrachtungen waren. ${ }^{13}$ Ebenso wenig konnte ich auf die lange Zeit vorherrschende strukturalistische Interpretation des Form-Bedeutungszusammenhangs, wie sie sich z.B. in Saussure (1967) findet, eingehen (siehe dazu auch Lepschy 1969). Mit großem Einfluss auf die Diskussion des Verhältnisses von Syntax und Semantik war auch die von Morris stammende semiotische Unterteilung in Syntaktik, Semantik und Pragmatik, die eine saubere Trennung von syntaktischen, semantischen und pragmatischen Phänomenen suggeriert, sie aber in der Realität nicht einlösen kann (vgl. Heringer/Strecker/Wimmer 1980: 113f, Lyons 1977: 114-119); auch diesen Punkt muss ich beiseite lassen. Es fehlt hier auch der Platz, die moderne Theoriebildung der letzten 50 Jahre nachzuzeichnen, die, dem jeweiligen Zeitgeist folgend, einmal die Semantik vollends aus der syntaktischen Analyse ausgeschlossen sehen wollte, ein anderes Mal sie zur Grundlage aller Syntax hochstilisierte. ${ }^{14}$

\section{Die BeiträGe}

Tor b e n T h r a n e , Handelshøjskolen in Århus, legt in seinem Beitrag „Hvorfor er sproget så svært at forstå når det er så let at forstå?“ (Warum ist die Sprache so schwer zu verstehen, wenn sie so leicht zu verstehen ist?) die Grundskizze einer informationstheorisch inspirierten, repräsentionellen Sprachtheorie vor. Die Bedeutungsebene wird bei Thrane als Repräsentationssystem erster Ordnung modelliert, das abstraktive (Modelle von) Situationen repräsentiert. Sprache im engeren Sinne hingegen wird als Repräsentationssystem zweiter Ordnung angesehen, in dem sprachliche Strukturen, v.a. syntaktische, repräsentiert werden. Die Interfaceproblematik stellt sich in dieser Theorie zweifach: einmal die Überführung von Repräsentationen erster Ordnung (Situationsre präsentationen, also „Bedeutungen“, in gewissem Sinn) in die der zweiten (sprachlich strukturierte Ausdrücke) bei der Produktion, bzw. umgekehrt bei der Rezeption, und einmal die wechselseitige Überführung von Daten erster Ordnung in die kognitiven Module (Verstehen) und umgekehrt (Produzieren).

Torben Thrane weist ausdrücklich auf das von mir oben eingeführte Schisma zwischen dem formellen und funktionalistischen Paradigma hin (autonome Syntax erwähnt er nicht ausdrücklich, ich vermute deshalb, weil er im Vorneherein die syntaktische Komponente, die ja Bestandteil des Repräsentationssystems zweiter Ordnung ist, in großen Teilen als autonom ansieht), kann sich aber keinem der beiden Lager vorbehaltlos anschließen, was wohl der Architektur seiner Theorie, insbesondere seiner Konzeption der Form-Bedeutungsschnittstelle $\mathrm{zu}$ danken ist. Torben Thrane diskutiert die repräsentationelle These von Sprache am Problembereich Argumentselektion, hierunter thematische Rollen sowie syntaktische Weglassbarkeit von Argumenten, wobei es 
ihm gelingt, miteinander konkurrierende grammatische Theorien wie G\&B, GPSG, HPSG oder LFG gleichermaßen auf seine informationstheoretische Konzeption der syntaktischen Komponente zu beziehen. Thranes Skizze einer informationstheoretisch en Sprachtheorie wäre sicher in vielen Punkten noch zu konkretisieren und empirisch zu untermauern, sie ist jedoch visionär und breit genug, wichtige und avancierte Zweige der formalen Syntaxforschung zu integrieren und sie im Wesentlichen als Notationsvarianten erscheinen zu lassen.

Hubert Ha ider, Universität Salzburg, stellt sich, ebenso wie Thrane, eine Frage: „Wie viel Syntax braucht die Semantik, und wie viel Semantik enthält die Syntax?“. Nicht ganz unähnlich meiner dreifachen Gegenüberstellung kompositional - funktional autonom, die ich einleitend begründet habe, legt Haider eine Zweiteilung zugrunde, die allerdings anders konzipiert ist. Haiders Version einer autonomen Syntax liefert eine hierarchisch und dependentiell strukturierte Oberflächensyntax, deren sich die Semantik zwar bedienen kann, die aber im Grunde eigengesetzlich aufgebaut ist. Haiders Konzeption einer kompositionalen Syntax, der Gegenpol, ist identisch mit meiner Charakterisierung, wogegen Haider jedoch kompositionale Syntax als repräsentativ für die Generative Grammatik ansieht. Mit Recht, wenn man die Logische Form LF, eine syntaktische Repräsentationsebene der Generativen Grammatik, die zwischen der Oberflächenstruktur und der semantischen Interpretation vermitteln soll, als hinreichend und adäquat genug strukturiert ansieht, um den Anforderungen einer kompositionalen Semantik zu genügen. Ich glaube, ich bin nicht der einzige, der hier Zweifel hat. Am Beispiel verschiedener Datenbereiche, der Adverbialsyntax, der Negation sowie der Argumentstruktur, demonstriert Haider, dass syntaktische und semantische Strukturierung natürlich oft parallel laufen, die Syntax allerdings ebenso häufig ihren eigenen Prinzipien folgt. In diesen Fällen ist es die Semantik, die „sich fügen“ muss.

Hans Arndt, Universität Aarhus, widmet sich dem „Beitrag der Syntax zur Bedeutung“ („The Contribution of Syntax to meaning“). Ausgehend von verschiedenen Typen von Bedeutung wie lexikalische Bedeutung, kollokationelle Bedeutung, syntaktische Bedeutung und schließlich pragmatische Bedeutung konzentriert sich Arndt zunächst auf die verschiedenen grammatischen Mittel, mit denen syntaktische Bedeutung zum Ausdruck gebracht werden kann (z.B: Wortfolge, Partikeln oder Prosodie). Seine Diskussion der syntaktischen Relationen wie die der Subjekt-PrädikatRelation führt ihn zu einer kritischen Sichtung der semantischen Rollen, die oft als Kandidaten für die Bedeutungsseite syntaktischer Funktionen betrachtet werden. Arndts Skepsis gegenüber dem schwer begrenzbaren System von semantischen Rollen hat deutliche Parallelen mit Thorben Thranes Beitrag zu diesem Heft. Beide ziehen aber verschiedene Schlüsse. Arndt sieht die Syntax letztendlich dem kompositionalen Prinzip verpflichtet, indem er die komplexe Syntax z.B. intransitiver, transitiver und ditransitiver Sätze sowie Konstruktionen mit eingebetteten Infinitiven und resultativer Konstruktionen semantisch als mehrfache und verschachtelte Prädikationen auffasst, die sich in der Syntax systematisch in wiederkehrenden semantischen Rollen sowie konstruktionsmässiger Gemeinsamkeiten niederschlagen. 
Per Aage Brandt, Universität Aarhus, hat seinen Beitrag überschrieben mit „Betydning og grammatik - i et semiotisk perspektiv“ (Bedeutung und Grammatik - in einer semiotischen Perspektive). Per Aage Brandt legt seinen Betrachtungen eine phänomenologische Sicht auf Sprache zugrunde, die auf dem beim Sprachbenutzer vorhandenen Sprachwissen sowie seiner Fertigkeit, mit einer robusten grammatischen Intuition Bedeutungen $\mathrm{zu}$ verstehen und sie $\mathrm{zu}$ produzieren, aufbaut. Dieses Sprachbewusstsein, das nach Brandt auch im Fremdsprachenunterricht zum Tragen kommt (nämlich dann, wenn pädagogisch aufbereitete Grammatiken vom Lerner unmittelbar als einsichtig empfunden werden), ist ein legitimer Prüfstein und lohnender Untersuchungsgegenstand für die grammatische und syntaktische Forschung. Diese einfache „online-Syntax“ demonstriert Brandt an einer Reihe einfacher Verknüpfungsoperationen, die er in einer stemmatischen, von Tesnière inspirierten Darstellung exemplifiziert. Der Beitrag schließt ab mit einer exemplarischen Analyse einer Zeitungsmeldung aus einer deutschen Tageszeitung.

Per Bærentzen, Universität Aarhus, untersucht in seinem Beitrag „Formale und semantische Unschärfen in vielgliedrigen Verbalkomplexen. Der Ersatzinfinitiv und anderes“. Bærentzen formuliert einleitend in Anlehnung an Bech die Prinzipien der Rektionsverhältnisse im deutschen Verbalkomplex sowie drei Typen von Abweichungen davon. Bærentzen legt dann eine ausführliche Analyse von abweichenden Strukturen wie Ersatzinfinitiv in drei germanischen Sprachen (Deutsch, Dänisch und Afrikaans) vor. Solche Abweichungen vom Grundprinzip resultieren nach Bærentzen in „semantischen Unschärfen“, die sich v.a. in der Temporalsemantik komplexer verbaler Konstituenten niederschlagen („defektive Temporalkonstruktionen“). Eine semantische Unschärfe liegt nach Bærentzen z.B. dann vor, wenn die im zweigliedrigen temporalen Verbalkomplex diktierten Rektionsverhältnisse (z.B. hab-PRÄS V/Part. Perf. habe gekonnt) im vielgliedrigen Syntagma ausser Kraft gesetzt sind (habe ... können), weil „[...] die Vergangenheit allein durch das Auxiliar [...] ausgedrückt wird [...]“ (Bærentzen, in diesem Band). Bærentzen schließt seinen Beitrag ab mit einer näheren Diskussion des leidigen Problem des deutschen Ersatzinfinitivs.

\section{Zum ABsChLUSS}

Die hier vorliegende Sondernummer von Tidsskrift for Sprogforskning sammelt die - stark überarbeiteten - Vorträge, die anlässlich des 4. Forschungskolloquiums Sprache am 27. November 2003 an der Staatsbibliothek in Århus gehalten wurden. Ich formulierte seinerzeit eine kurze Beschreibung des Themas „Syntax und Bedeutung“, die den inhaltlichen Rahmen des Kolloquiums abstecken - und eine gewisse gemeinsame Ausrichtung der Vorträge fördern sollte. Hier der Wortlaut:

„Syntax kann verstanden werden als System von Kombinationsregeln, welche die Sprachzeichen auf solche Weise verbinden, dass sie grammatisch korrekte komplexe Ausdrücke bilden (nicht ungleich einer Syntax einer künstlichen Sprache, z.B. einer Logiksprache). 
Diese radikale Art und Weise, Syntax zu betrachten, war allerdings nie besonders anziehend und attraktiv für praktische Syntaktiker, indem deren Beschreibungen trotz des formalen Dogmas der Syntax immer und in hohem Grad mit Entitäten aus der Bedeutungssphäre operiert haben wie z.B. Kasusrollen, Referenzmöglichkeiten von Wortklassen oder semantische Modifikationsrelationen zwischen Satzelementen im Satzverband, nur um einige wenige zu nennen. Hierzu kommen eine Reihe von Text- und Kontextelementen wie Thema-Rhema (z.B. in Studien zur Wortstellung), satzüberschreitende anaphorische Relationen und die sprechakttheoretische Einbettung von Sätzen in größere kommunikative Zusammenhänge (z.B. im Rahmen der Analyse von Satztypen oder von Untersuchungen des syntaktischen Verhaltens von Diskurspartikeln).

In vielen dieser Untersuchungen wird allerdings das Verhältnis von Bedeutung und den beobachteten syntaktischen Zügen der Ausdrücke selten explizit thematisiert. Ist dies einer Vorstellung von Kompositionalität zu schulden, wo Bedeutung parallel mit den syntaktischen Ausdrücken aufgebaut wird, wie es radikal in der Montague-Grammatik durchgeführt ist? Gibt es vielleicht einen grundlegenden Mangel bei syntaktischen Regeln, die alleine - ohne Rekurs auf semantische Kategorien - nicht die syntaktisch korrekte Form garantieren können? Oder findet sich ein instinktives Gefühl beim Syntaktiker, dass syntaktische Gegenstände und Regeln erst dann Gegenstände mit ,Fleisch und Blut' werden, wenn man ihnen Bedeutung oder ,Funktion' anheftet? Oder etwas ganz anderes?

Diese Interface-Problematik ist ziemlich entscheidend, weil sie sich darum dreht, wie syntaktische Form, d.h. formale Regeln auf Satzniveau, zum Ausdruck (oder zur Dekodierung) von Bedeutung benutzt werden. Darüber hinaus spiegeln Interface-Konzeptionen zwei verschiedene Haltungen gegenüber Sprache wider, die funktionelle Haltung, die ihren Ausgangspunkt in den kommunikativen Aufgaben der Sprache sucht und danach fragt, wie syntaktische Regeln beschaffen sein müssen, diese Aufgaben erfüllen zu können; der Autonomiehypothese zufolge ist die Syntax ein autonomes System, dessen Regeln unabhängig von bedeutungsrelatierten Einheiten und Sprachfunktionen funktioniert. Beide Sichtweisen auf das Syntax-SemantikInterface kommen in modernen syntaktischen Analysen zum Tragen, oft gemischt und selten direkt problematisiert."

Ich denke, die hier vorgelegten Beiträge greifen anhand von konkreten Analysen viele der oben geschilderten Problematiken auf. Und es ist mein Wunsch, dass der Leser dieses Sonderheftes der Tidsskrift for Sprogforskning einen Eindruck davon bekommt, wie relevant und vielschichtig - und spannend! - das Form-Bedeutungsverhältnis in der 
Sprache ist. Viel Vergnügen beim Lesen!

\section{LITERATUR}

Andersen, Paul Kent (1991), A new look at the passive, Frankfurt/M. etc.: Peter Lang.

Arens, Hans (1969), Sprachwissenschaft. Der Gang ihrer Entwicklung von der Antike bis zur Gegenwart, Freiburg, München: Karl Alber.

Bartsch, Renate, Theo Vennemann (1980), "4. Sprachtheorie“, in: Althaus, Hans Peter, Helmut Henne, Herbert Ernst Wiegand (Hgg.) (1980), Lexikon der Germanistischen Linguistik, Bd. 1, Tübingen: Niemeyer, pp. $57-82$.

Bierwisch, Manfred (1965), „Eine Hierarchie syntaktisch-semantischer Merkmale“, in: Syntaktische Studien, Berlin: Akdemie-Verlag, pp. 29 - 86 (= Studia Grammatica, 5).

Borsley, Robert D. (1997), Syntax-Theorie. Ein zusammengefasster Zugang, Tübingen: Niemeyer.

Bußmann, Hadumod (2002), Lexikon der Sprachwissenschaft, Stuttgart: Kröner.

Cann, Ronnie (1993), Formal semantics. An introduction, Cambridge: Cambridge University Press.

Chomsky, Noam (1969), Aspekte der Syntax-Theorie, Frankfurt/M., Berlin: Suhrkamp und Akademie Verlag.

Chomsky, Noam (1981), Regeln und Repräsentationen, Frankfurt/M.: Suhrkamp.

Chierchia, Gennaro, Sally McConnell-Ginet (1990), An Introduction to Semantics, Cambridge/Mass., London: MIT Press.

Dowty, David R., Robert E. Wall, Stanley Peters (1981), Introduction to Montague semantics, Dordrecht: Reidel.

Dudengrammatik, s. Dudenredaktion ...

Dudenredaktion (1998), Duden. Grammatik der deutschen Gegenwartsprache, Mannheim etc.: Dudenverlag.

Eisenberg, Peter (1999), Grundriss der deutschen Grammatik, Bd. 2, Der Satz, Stuttgart, Weimar: Metzler.

Enç, Mürvet (1988), „The syntax-semantics interface“, in: Newmeyer, Frederick J. (ed.) (1988), Linguistics: The Cambridge Survey, Vol. 1, Linguistic Theory: Foundations, Cambridge etc.: Cambridge University Press, pp. 239 - 254.

Engel, Ulrich (1988), Deutsche Grammatik, Heidelberg: Julius Groos.

Engerer, Volkmar (1999), "Phasenverbkomplemente", Studia Germanica Universitatis Vesprimiensis, 3/2 (1999), pp. 145 - 159.

Engerer, Volkmar (2001), „Austin - Searle - Haugaard!? En sproghandlingsteoretisk analyse af Jacob Haugaards valgløfter", in: Engerer, Volkmar (udg./Hg.) (2001), Sproghandlingsteori/ Sprechakttheorie, Århus: Germanistisches Institut der Universität Aarhus, pp. 119 - 151 (= Augias, Nr. 56-59).

Fanselow, Gisbert, Sascha W. Felix (1990), Sprachtheorie, Bd. 1, Grundlagen und Zielsetzungen, Tübingen: Francke.

Helbig, Gerhard (1988), Entwicklung der Sprachwissenschaft seit 1970, Leipzig: Bibliographisches Institut.

Heringer, Hans Jürgen, Bruno Strecker, Rainer Wimmer (1980), Syntax. Fragen - Lösungen - Alternativen, München: Wilhelm Fink.

Joos, Martin (ed.) (1968), Readings in Linguistics I. The Development of Descriptive Linguistics in America 1925-56, Chicago, London: University of Chicago Press. 
Kempson, Ruth (1977), Semantic Theory, Cambridge etc.: Cambridge University Press. Klaiman, M. H. (1991), Grammatical voice, Cambridge etc.: Cambridge University Press.

Lepschy, Giulio C. (1969), Die strukturale Sprachwissenschaft. Eine Einführung, München: Nymphenburger.

Leuninger, Helen (1979), Reflexionen über die Universalgrammatik, Frankfurt/M.: Suhrkamp.

Lewis, David (1976), „General semantics“, in: Partee, Barbara H. (ed.) (1976), Montague Grammar, New York, San Francisco, London: Academic Press, pp. 1 - 50.

Lohnstein, Horst (1996), Formale Semantik und natürliche Sprache, Opladen: Westdeutscher Verlag.

Lyons, John (1977), Semantics, Vol. 1, Cambridge: Cambridge University Press.

Maas, Utz (1979), Grundkurs Sprachwissenschaft, Teil 1, Grammatiktheorie (die herrschende Lehre), Frankfurt/M.: Syndikat.

Matthews, P. H. (1981), Syntax, Cambridge etc.: Cambridge University Press.

Pinker, Steven (1996), Der Sprachinstinkt. Wie der Geist die Sprache bildet, München: Kindler.

Saussure, Ferdinand de (1967), Grundfragen der allgemeinen Sprachwissenschaft, Berlin: de Gruyter.

Schmidt, Ulrich A. (1987), Impersonalia, Diathesen und die deutsche Satzgliedstellung, Bochum: Brockmeyer.

Schoenthal, Gisela (1976), Das Passiv in der deutschen Standardsprache. Darstellung in der neueren Grammatiktheorie und Verwendung in Texten gesprochener Sprache, München: Max Hueber.

Searle, John R. (1971), Sprechakte. Ein sprachphilosophischer Essay, Frankfurt/M.: Suhrkamp.

Searle, John R. (1982), "Eine Taxonomie illokutionärer Akte", in: Searle, John R. (1982), Ausdruck und Bedeutung, Frankfurt/M.: Suhrkamp, pp. 17 - 50.

Searle, John R., Daniel Vanderveken (1985), Foundations of Illocutionary Logic, Cambridge: Cambridge University Press.

Seebaß, Gottfried (1981), Das Problem von Sprache und Denken, Frankfurt/M.: Suhrkamp.

Serébrennikow, B. A. (Leitung des Autorenkollektivs) (1975), Allgemeine Sprachwissenschaft, Bd. 2, Die innere Struktur der Sprache, München, Salzburg: Wilhelm Fink.

Seuren, Pieter A. M. (1996), Semantic Syntax, Oxford: Blackwell.

Stechow, Arnim von, Wolfgang Sternefeld (1988), Bausteine syntaktischen Wissens. Ein Lehrbuch der generativen Grammatik, Opladen: Westdeutscher Verlag.

Stegmüller, Wolfgang (1987), Hauptströmungen der Gegenwartsphilosophie, Bd. 2, Stuttgart: Kröner.

Vanderveken, Daniel (1990/1991), Meaning and speech acts, 2 vols., Cambridge: Cambridge University Press.

Vikner, Sten (2004), „Nødvendigheden af en formel tilgang til sprogvidenskab“, Humaniora, 2 (2004), pp. 13-16. 
Engerer, Einleitung

\section{ANMERKUNGEN}

1 Ausführliche Diskussionen des Grammatikbegriffs in der Sprachtheorie finden sich in Bartsch/ Vennemann (1980: 58-63) sowie Serébrennikow (1975: 160-168). Eine, obschon etwas veraltete, aber immer noch sehr gründliche Einführung in die semantische Theorie ist Kempson (1977).

2 Der Ausdruck "Funktionale Grammatiken" ist dem Lexikon der Sprachwissenschaft (Bußmann 2002: 229f) entnommen. Dort werden funktionale Grammatiken als Alternativen „[...] $\mathrm{zu}$ (post)strukturalistischen Ansätzen, die sprachliche Phänomene formal (z.B. autonom in der Syntax) zu erfassen versuchen“, dargestellt (Bußmann 2002: 230). Ich möchte, im Gegensatz zu Bußmann (2002), zwischen „formal“ und „formell“ unterscheiden. „Formell“ gebrauche ich in Anlehnung an Vikner, wo das dänische „formel“ Verwendung findet, gleichbedeutend mit ,formbezogen', wogegen „formal“ die Benutzung logiksprachlicher und mathematischer Elemente und Hilfsmittel indiziert. In diesem Sinne können formelle Grammatiktheorien sowohl formal als auch informal sein, ebenso finden sich funktionale grammatische Theorien in formalen und informalen Ausfertigungen.

3 Weitere Verschiebungen und Bewegungsprozesse, von denen z.B. das finite Modalverb vil betroffen ist (sein Platz ist „eigentlich“ beim infiniten Hauptverb bo, das es regiert), behandle ich hier nicht. Mehr über die Bewegungsmetapher sowie repräsentationelle Alternativen in der generativen Grammatik findet sich in Stechow/Sternefeld (1988).

4 Ich brauche hier "Formalismus" als substantivische Ableitung von "formell" im Sinne von 'formbezogen'. „Formalismus“ ist leider zweideutig im Sinne des Bezugs auf „formell“ oder „formal“, weil eine spezielle substantivische Ableitungsform von „formell“ im Deutschen nicht zur Verfügung steht. Ich muss mich also im Weiteren auf den Kontext verlassen - und werde dort, wo ein Missverständnis fatale Folgen haben könnte, explizit auf die intendierte Variante hinweisen.

5 Leuninger (1979: 26) macht darauf aufmerksam, dass die These von der Autonomie der Syntax schon in den frühen Arbeiten von Chomsky enthalten gewesen sei. Ich habe das nicht weiter nachgeprüft, s.a. die dort gegebenen Literaturhinweise.

6 So gesehen ist es bestimmt kein Zufall, dass Vikner (2004) ausschließlich Beispiele aus dem Bereich der Syntax (Wortstellung) und der Phonologie (systematische phonologische Reduktionen der Flexionsendungen mit $-e$ bei dänischen Adjektiven (dumm-e) und Substantiven (blad-e) wählt, um die Vorzüge (und die Überlegenheit) des formellen Zugangs zu demonstrieren.

7 Vgl. die noch immer klassische Einführung in die Montague-Grammatik von Dowty/Wall/ Peters (1981). Als Beispiel für eine ernstzunehmende Kritik an der „Bedeutungsfeindlichkeit“ der generativen Theorie führen Fanselow/Felix (1990: 86) Seebaß (1981) auf, ohne allerdings Seitenzahlen anzugeben. Mir war es deshalb nicht möglich, diesem Hinweis nachzugehen.

$8 \mathrm{Zu}$ einer ausführlichen Besprechungen der Kontrolleigenschaften deutscher und englischer Verben im Rahmen der GB- und HPSG-Theorie siehe Borsley (1997: 271-291). Eisenberg erörtert Kontrollprobleme im Kapitel über Infinitivkonstruktionen (Eisenberg 1999: 339ff). Kontrolle bei Phasenverben ist u.a. Gegenstand von Engerer (1999).

9 Versprechen und Bitten gehören in der Terminologie Searles zu den kommissiven bzw. den direktiven Sprechakten, vgl. dazu Searle (1971), wo v.a. Versprechen ausführlich behandelt werden, sowie Searle (1982), wo die entsprechenden Sprechaktklassen erstmals entwickelt werden. Auch in den formalen Weiterentwicklungen der Sprechakttheorie (vgl. z.B. Searle/ Vanderveken 1985 sowie Vanderveken 1990/1991) spielen Versprechen und Bitten eine ausgezeichnete Rolle. Versprechen im Sinne von Wahlversprechen untersucht Engerer (2001).

10 Zum Passiv im Deutschen siehe auch Schoenthal (1976) und Schmidt (1987). Allgemein zum Passiv und Diathese vgl. Klaiman (1991) sowie Andersen (1991). Enç (1988: 242) demonstriert ganz kurz die Lösung im G\&B-Format, die ebenso auf einer Ableitungsbeziehung zwischen Aktiv- und Passivsatz beruht.

11 Das Prinzip der Kompositionalität wird Gottlob Frege zugeschrieben. Es liegt in der Tradi- 
tion der Montague-Grammatik fast allen formalen semantischen Theorien zugrunde. Eine Formulierung stammt von Stegmüller, der es unter dem „Grundsatz der semantischen Funktionalität" bei seinem Durchgang der Prinzipien der Montague-Grammatik so ausdrückt: „Bedeutung sowie Denotat jedes wohlgeformten komplexen Ausdruckes A ist eine eindeutig bestimmte Funktion der Bedeutungen bzw. der Denotate der wohlgeformten Teilausdrücke von A." (Stegmüller 1987: 54). Linguistisch mehr angepasste Formulierungen des Kompositionalitätsprinzips finden sich in allen modernen Einführungen zur formalen Semantik, z.B. in Lohnstein (1996: 54): „Die Bedeutung eines komplexen Ausdrucks lässt sich aus der Bedeutung der Einzelausdrücke und der Struktur des Gesamtausdrucks berechnen.“ Ähnlich Cann (1993: 2ff) und Chierchia/McConnell-Ginet (1990).

12 In etwas anderer Form schlägt sich die kompositionale Sicht auf die Syntax auch in Matthews (1981: 3) nieder. Syntaktische Konstruktionsbeziehungen sind hier Bedeutungsbeziehungen, die nicht durch pure Distribution erschöpft werden können. Dies hat u.a. zur Konsequenz, dass syntaktische Konstruktionsunterschiede (immer?) Bedeutungsunterschieden entsprechen.

13 Viele Texte in Arens (1969), die von der Antike über das Mittelalter bis zur Neuzeit spannen, bezeugen die Zentralität des Form-Bedeutungsverhältnisses.

14 Der Amerikanische Strukturalismus („asemantischer Strukturalismus“, vgl. Heringer/Strecker/ Wimmer 1980: 116) ist wohl ein gutes Beispiel für den Versuch einer bedeutungsentleerten linguistischen Formwissenschaft (s. die gesammelten Artikel in Joos 1968 sowie Maas 1979), in gewissem Sinne auch behavioristische Strömungen Mitte des 20. Jahrhunderts (s. z.B. das Kapitel „Bevaviorist semantics“ in Lyons 1977: 120-137). Eine erste Annäherung semantischer und syntaktischer Denkweisen zeigt schon der frühe Aufsatz „Eine Hierarchie syntaktischsemantischer Merkmale“ von Manfred Bierwisch (Bierwisch 1965), eine Entwicklung, die mit dem Primat der Semantik in der Generativen Semantik kulminiert (s. Helbig 1988: 111ff sowie Pieter A. M. Seurens Semantic Syntax (Seuren 1996) als einer der wenigen modernen Nachfolger, welche der Generativen Semantik wohl auch keine Renaissance bereiten können). Heute scheint es mir, dass Syntaktiker und Semantiker mehr oder weniger ihr Eigenleben führen - eigentlich schade. 